\title{
Explaining the socio-economic gradient in child outcomes: the inter-generational transmission of cognitive skills
}

\section{Claire Crawford}

Institute for Fiscal Studies and Institute of Education, University of London

claire c@ifs.org.uk

\section{Alissa Goodman}

Institute for Fiscal Studies

\section{Robert Joyce}

Institute for Fiscal Studies

(Received July 2010 Revised November 2010)

\section{Abstract}

Papers in this Special Issue and elsewhere consistently find a strong relationship between children's cognitive abilities and their parents' socio-economic position (SEP). Most studies seeking to explain the paths through which SEP affects cognitive skills suffer from a potentially serious omitted variables problem, as they are unable to account for an important determinant of children's cognitive abilities, namely parental cognitive ability. A range of econometric strategies have been employed to overcome this issue, but in this paper, we adopt the very simple (but rarely available) route of using data that includes a range of parental characteristics measured during the parents' childhood, such as parental cognitive ability and social skills. In line with previous work on the intergenerational transmission of cognitive skills, we find that parental cognitive ability is a significant predictor of children's cognitive ability; moreover, it explains one sixth of the socio-economic gap in those skills, even after controlling for a rich set of demographic, attitudinal and behavioural factors. Despite the importance of parental cognitive ability in explaining children's cognitive ability, however, the additional parental characteristics we examine here do not alter our impression of the relative importance of other factors in explaining the socio-economic gap in cognitive skills. This is reassuring for studies that are unable to control for such characteristics.

JEL codes: I20, I32

Key words: cognitive skills, intergenerational transmission, socio-economic gap

\section{Background and motivation}

Papers in this Special Issue and elsewhere have consistently found a strong relationship between a child's cognitive skills and his or her parents' socioeconomic position (henceforth SEP). Previous research on this topic has sought to understand both: i) whether it is parents' SEP which influences children's cognitive test scores, or other correlated factors (Dahl and Lochner 2008; Brooks-Gunn and Duncan 1997; Mayer 1997), and ii), the transmission mechanisms through which parents' SEP might influence test scores (taking i) as given) (Guo and Harris 2000; Davis-Kean 2005; Jean Yeung et al 2008, plus papers in this issue). Parents are likely to have many characteristics that are correlated both with SEP and with their children's cognitive outcomes. Many of these characteristics are rarely observed, such as social skills, other personality traits and (at least in UK data) parental 
cognitive ability. This presents a potentially serious omitted variables problem with considerable policy implications. By omitting important variables from models relating parents' SEP to child outcomes, researchers risk both overstating the importance of parental SEP in determining children's cognitive outcomes, and falsely pinpointing specific transmission mechanisms between them. Such mistakes could lead to misguided policy recommendations, both in terms of how much raising the living standards of children growing up in poverty is likely to improve their cognitive development (and hence, through various channels, their own living standards as adults); and indeed whether specific interventions, such as those that target particular hypothesised transmission mechanisms, are likely to be effective.

Such latent factors may play a large role in explaining why children from poorer families have worse cognitive outcomes than children from richer families: simply put, more able parents are likely both to be able to command higher incomes in the labour market, and might also raise more cognitively developed children for other reasons, for example through better parenting skills, a greater preference for educational investments in their children, or due to some direct, more genetic link in cognitive ability between parents and their offspring. Given the growing literature on the importance of an individual's non-cognitive skills for a wide range of economic and social outcomes (see, for example, Blanden et al 2007; Heckman et al 2006; Carneiro et al 2007), a similar line of argument applies to the omission of these types of parental characteristics in such analyses.

There are a significant number of studies employing a range of econometric strategies to tackle the problem of omitted variables in estimating the relationship between parental income and a number of child outcomes, among which measures of children's educational attainment feature heavily and measures of cognitive development feature occasionally. However, there are few papers, to our knowledge, that attempt to identify the potential transmission mechanisms through which income may affect these outcomes in a similarly robust framework.

Typical among the approaches taken in the studies that do attempt to address the issue of correlated unobservable characteristics are those that adopt some sort of experimental or quasi- experimental approach (Duflo 2000; Mayer 2007; Duncan Morris and Rodrigues 2006); those that make use of instrumental variables (IV) techniques (Shea 2000; Dahl and Lochner 2008); and those that use sibling differences or other fixed effects-type methodologies (Blau 1999; Duncan et al 1998; Levy and Duncan 2000; Ermisch et al 2002). While these approaches share the advantage that they can, under certain assumptions, identify the causal effects of specific observed variables in the absence of any data on unobserved characteristics, they also suffer from obvious drawbacks. First, the identifying assumptions are often problematic, such as exogeneity of the instrument in IV approaches and strict exogeneity in fixed-effects studies (which rules out 'feedback' effects within families in the case of sibling difference models). Second, even if the identifying assumptions are valid, we learn nothing about the key sources of the omitted variables bias: we only consistently estimate the causal effects of things we observe, but the things we do not observe may be at least as important (in terms of explaining the outcome of interest) and thus of immense policy relevance.

Few papers follow our approach, which is to take the very simple (but rarely available) route of utilising data in which some of these variables are observable. Here, we make use of a dataset - the British Cohort Study (BCS) - that provides detailed information on cognitive ability, SEP, family background characteristics, health, social skills and attitudes and behaviours across two generations within the same families. We therefore observe the cognitive ability of (one of) the parents of each child in our sample, as well as other parental characteristics that are often unobservable to the researcher looking at the determinants of children's cognitive skills. The contribution of this paper is not to identify the causal effect of SEP on children's cognitive abilities, but rather to investigate how this additional information changes our understanding of what matters for cognitive development.

Like the other papers in this series, we emphasise that our analysis is not causal: we cannot rule out endogeneity due to omitted variables or reverse causation. Instead, we aim to identify possible transmission mechanisms between SEP and cognitive skills that can rarely be investigated in studies of this kind, thus highlighting areas where future experimental research aimed at identifying causal effects may be best targeted. Crucially, we 
also aim to supplement the other papers in this series by assessing whether information about additional parental characteristics, such as cognitive ability, might change their conclusions about which transmission mechanisms matter. To do so, we compare the results obtained from regressions of a child's cognitive ability on a wide range of explanatory variables with and without including often unobserved parental characteristics in the model. We also use these estimates to 'decompose' the SEP gap in cognitive skills into the contributions of various groups of factors, again comparing the results obtained with and without accounting for the parental characteristics that are often unobservable to the researcher.

By doing this we also contribute to a wider literature on the intergenerational transmission of cognitive abilities, in which the correlation of cognitive ability between parents and children is well documented (for recent evidence, see Anger and Heineck 2010; Black et al 2009; Björklund et al 2009). Genetic inheritance is one obvious potential explanation for the observed correlation in ability across generations, but the extent of its explanatory role is contested: this is a complex literature spanning a number of disciplines, and one in which the simplistic dichotomy between 'nature vs nurture' in explaining child development is increasingly recognised as overly simplistic and obsolete (see Cunha and Heckman 2007). Decomposing environmental from genetic effects is complicated by the likelihood that the environments people choose (or parents choose on their behalf) are ones which complement their genetic endowments (Dickens and Flynn 2001; Loehlin 2002; Rowe and Rodgers 2002; Dickens and Flynn 2002).

Complementing this literature, we find a very strong correlation between cognitive ability across generations, as well as a strong relationship between family socio-economic status and cognitive ability in both generations. Our decompositions suggest that often unobserved information on parental cognitive ability, social skills and attitudes to education, explains almost one quarter of the gap in cognitive test scores between high and low SEP children. This mostly reflects the importance of parental cognitive ability, which accounts for 16 percent of the gap after controlling for a wide range of mechanisms through which cognitive ability may be passed across generations (for example, differences in the home learning environment that parents provide for their children), and 50 percent of the gap if we do not include such factors. As the importance of parental ability remains substantial after controlling for so many of the mechanisms through which it may plausibly affect child's cognitive ability, one possibility - though not the only one - is that genetic transmission of cognitive skills is significant. Reassuringly for previous studies, however, we find that accounting for these additional parental characteristics does not hugely alter our impression of which factors observed during the current generation are important for cognitive skills (attitudes towards education and social skills remain the most important predictors).

This paper now proceeds as follows: Section 2 provides a brief description of the BCS data and some summary statistics. Section 3 describes the omitted variable problem that studies of children's cognitive skills typically face when key parental characteristics are unobserved, and illustrates the likely extent of this problem using some simple analysis of the BCS data, which includes many of these characteristics. Sections 4 and 5 present our main analysis, starting with our regression results (in Section 4), which are then used as the basis for a decomposition analysis of the SEP gap in children's cognitive skills (in Section 5). Section 6 concludes.

\section{Data}

The British Cohort Study (BCS) sampled all people born in Great Britain in a particular week in April 1970 and has surveyed them at various points throughout their lives. ${ }^{1}$ We have data from seven waves: at the cohort members' birth, and at ages 5 , $10,16,26,29$ and 34 . Importantly for our purposes, the age 34 wave (in 2004) randomly selected half of the cohort members (henceforth CMs) who lived with their natural or adopted children for an additional battery of questions about those children. ${ }^{2}$ This means that we have rich measures of cognitive ability, social skills, attitudes and behaviours and family background characteristics across two generations within the same family. Note, however, that while the CMs are all the same age, the children of the CMs were all interviewed at the same time and are therefore of different ages. This is an important issue to which we return later.

Our main outcome of interest is the cohort member's child's cognitive ability, as measured in 
2004 using the British Ability Scales (BAS). ${ }^{3}$ Children aged 3 to 5 were tested on vocabulary and early number concepts; those aged 6 and over were tested on word reading, spelling and number skills.

We would like to age-normalise these test scores to take account of the fact that cognitive performance tends to increase with age. This is typically achieved by using the residuals from a regression of test scores on age. The problem with this procedure in our data is that, as explained, the age of the child is collinear with the age of one of their parents (the cohort member) at the child's birth, which is itself correlated with a wide range of possible determinants of cognitive skills (such as SEP and parental ability). Thus, a straightforward age-normalisation would effectively be 'partially normalising' with respect to these possible determinants of cognitive skills (because they are correlated with age). Clearly this could be undesirable, since it is precisely the impact of these variables on cognitive skills that we want to investigate. To avoid this problem whilst normalising for the actual 'effect' of age as best we can, we run the following two-step procedure when defining our outcome variable:

1) Estimate the equation: cognitive test score $=$ age' $\alpha+X^{\prime} \beta+u$

2) Estimate the 'residual': cognitive test score age' $\alpha$ by replacing $\alpha$ with its first stage estimate.

In the above, 'age' is a matrix of dummy variables capturing each possible age of the child in months, ' $X$ ' is a matrix of all other variables that we use in our full regression specification including a constant (see sections 4 and 5), $\alpha$ and $\beta$ are coefficient vectors, and $\mathrm{u}$ is a vector of error terms. The 'residual' (estimated in step 2) is our agenormalised score. This differs from a more standard age-normalisation, because we control for $X$ in the first stage. Having carried out the agenormalisation, we arrange the normalised scores into percentile ranks for our analysis. Note that we have repeated our analysis with test scores agenormalised in the standard way, and our results are extremely similar. In addition, as we emphasise later, our main conclusions also hold if we disaggregate the sample of children by age.

Our aim is to explain variation in this agenormalised measure of children's cognitive ability using not only information from the current generation (such as SEP and other family background characteristics, plus attitudes and behaviours of both the parents and their children ${ }^{4}$ ), but also information that is often unobserved from the CM's own childhood. The source of this often unobserved information is the first four waves of the BCS (from birth to age 16). Specifically, we make use of cognitive ability test scores at ages 5 and 10, social skills measures at ages 5 and 10 and a range of other information on attitudes and behaviours at ages 10 and 16 .

At age 5, the cognitive ability measure includes tests on vocabulary, copying designs, human figure drawing, and profile recognition. At age 10, it includes tests from the British Ability Scales (BAS), as well as additional tests of reading, vocabulary, writing, spelling, maths, copying, sentence formation and sequence recognition. At each age, we average across standardised scores from each test and split the sample of cohort members into cognitive ability quintiles on the basis of this average.

The social skills measures are based on mother reports of the Rutter behaviour scale ${ }^{5}$ at ages 5 and 10 and the Conners behaviour scale at age 10 (see Conners 1969). Other attitudinal information from the parents' childhood that we control for includes: measures of self-perceived ability, self-esteem and self-concept at ages 10 and 16; and attitudes at age 16 towards school, the prospect of post-compulsory education, and the importance of earning lots of money in the future. 6

We are also interested in the extent to which these factors can help to explain the gap in cognitive ability scores between children from rich and poor families. As described in Goodman, Gregg and Washbrook 2010, we follow the standard approach of using polychoric principal components analysis to construct an index of socio-economic position, using information on permanent family income, ${ }^{7}$ housing tenure, parental occupational class and an indicator of financial difficulty (the first principal component explains 39\% of the variation in these variables). Where there is missing or insufficient information on one or more of these dimensions (but not all of them), we use multiple imputation techniques to help construct the SEP index. The SEP gaps in each component of the SEP index are in Table A2 in our Supplementary Files.

Table 1 presents some summary statistics for various subsamples of the BCS cohort, from the original childhood cohort (column 1) to our estimation sample of the CMs' children in 2004 
(column 5). It shows that almost $50 \%$ of the original sample of $\mathrm{CMs}$ had dropped out of the survey by 2004 (compare columns 1 and 2). This attrition was non-random: the CMs who dropped out before 2004 were relatively more likely to be from low SEP backgrounds and of low cognitive ability, and slightly more likely to be male.

Since the CMs are the parents of the children we study, the parents are all the same age. ${ }^{8}$ Some of them will go on to have children, but had not done so by age 34 when surveyed. Therefore, those $\mathrm{CMs}$ with children in our sample are relatively likely to have characteristics that are not only correlated with having children (which follows trivially), but correlated with having children at a younger age: in our data, such characteristics include being female, less cognitively able, less well educated, and in a lower SEP group (compare columns 2 and 3 ). This aspect of the sample selection offsets some, but not all, of the over-sampling of high-SEP and high cognitive ability CMs caused by attrition (although clearly we cannot be sure that these two aspects of the sample selection act to 'offset' each other in terms of characteristics that we do not observe).

Column 4 additionally restricts our sample to the CMs who were included in the additional child surveys, and whose children completed the cognitive ability tests. We restrict our attention to 3 to 16 year-old children, since they were all assessed using the British Ability Scale, and the developmental scores for under-3s are not comparable. This leaves us with 3,416 children from 2,059 families as our main estimation sample (column 5). This age restriction again excludes children whose parents were older when they were born, with the same implications for the gender, SEP, cognitive ability and education balance of the parents of the children in our estimation sample.

\section{Parental ability and the omitted variable problem}

The other papers in this Special Issue describe how children growing up in poorer families have worse cognitive outcomes than children growing up in richer families, and seek to explain this association by considering factors observed in the current generation (such as the home-learning environment and parents' attitudes to education) which are correlated with both SEP and cognitive outcomes. The BCS allows us to extend this analysis back a generation by taking account of much more detailed information about one of the children's parents. 


\section{Table 1. BCS sample descriptive}

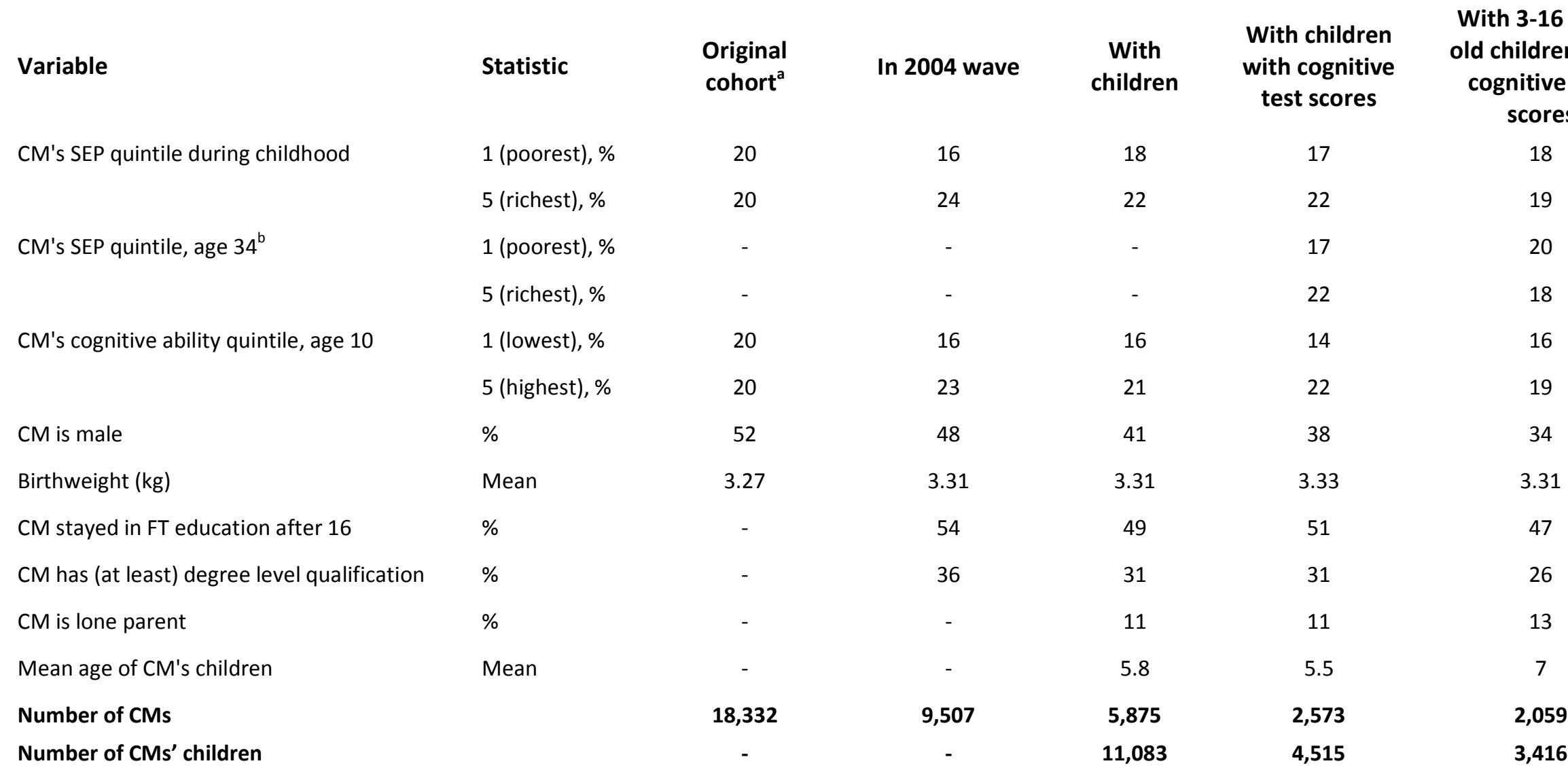

a) The 'original' cohort includes 1,409 CMs who were not surveyed in the first wave (at birth) but were surveyed later in childhood (at ages 5 , 10 or 16). It excludes 273 CMs who were surveyed as children but did not provide sufficient information for us to construct their SEP index.

b) Note that the age 34 SEP distribution from which we define SEP quintiles is the distribution of the CMs' children, since they are our estimation sample (not the CMs themselves). There are thus more CMs in the top quintile than in the bottom quintile in column 4 , since those in the top quintile have fewer children, on average. 
In particular, we have data on the performance of the CMs in cognitive tests when they were children. The potential significance of this additional information is large. Children's cognitive skills, parents' cognitive skills and SEP could all be causally related ${ }^{9}$ and are certainly correlated: more able parents are likely to command higher incomes in the labour market, and might also raise more cognitively developed children for other reasons, for example through better parenting skills. To the extent that the effects of parental ability on cognitive test scores are not perfectly captured by other factors observed in the current generation (for example, the quality of the home learning environment that they provide for their children), analysis of the link between children's cognitive skills and SEP has an omitted variable problem to contend with when parental ability is unobserved. ${ }^{10}$ Here, as background to our analysis, we document the extent of the association between parental cognitive ability, child cognitive skills and SEP in our sample.

Table 2 shows the extent of the intergenerational links in cognitive ability; or, to put it another way, the raw correlation between our outcome of interest (children's cognitive ability) and an explanatory variable that is not usually observable in most UK datasets (parental cognitive ability). We split both children and their parents into ability quintiles, creating 25 possible combinations of child and parental ability. ${ }^{11}$ The Table shows, for given parental ability quintiles, the percentage of children in each ability quintile (so the numbers in each row sum to 100 , subject to rounding). There is clear persistence of high and low cognitive abilities across generations: for example, of those children whose parent was in the lowest cognitive ability quintile at age $10,29 \%$ are in the lowest quintile themselves, with just $14 \%$ in the highest quintile. ${ }^{12}$

Table 2. Cognitive ability across generations

$\%$ from each parental ability quintile

$\downarrow$ Parent's cognitive ability quintile, age 10
Low
2
3
4
High

$\begin{array}{cc}\text { Low } & \mathbf{2} \\ 29 & 23 \\ 25 & 20 \\ 16 & 21 \\ 15 & 19 \\ 12 & 18\end{array}$

Child's cognitive ability quintile

$\begin{array}{cccc}\mathbf{2} & \mathbf{3} & \mathbf{4} & \text { High } \\ 23 & 19 & 14 & 14 \\ 20 & 19 & 17 & 19 \\ 21 & 22 & 22 & 19 \\ 19 & 19 & 23 & 24 \\ 18 & 20 & 23 & 27\end{array}$

Table 3. The association between parental ability and socio-economic position

$\%$ from each parental ability quintile

$\downarrow$ Parent's cognitive ability quintile, age 10
Low
2
3
4
High

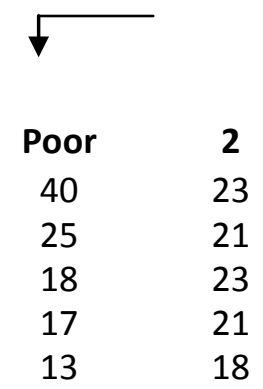

SEP quintile

$\begin{array}{ccc}3 & \mathbf{4} & \text { Rich } \\ 18 & 15 & 4 \\ 22 & 23 & 10 \\ 21 & 20 & 17 \\ 23 & 19 & 20 \\ 16 & 26 & 27\end{array}$

Furthermore, Table 3 shows that children growing up in high SEP families are far more likely to have cognitively able parents. This pattern is particularly striking at the bottom of the parental ability distribution: among children whose parents are in the bottom cognitive ability quintile, ten times as many are in the poorest SEP quintile (40\%) as in the richest (4\%). This suggests that, if parental ability is an important determinant of children's abilities, observing SEP but not parental ability could lead to erroneous conclusions about the 
determinants of cognitive skills (in the absence of a valid identification strategy).

Figure 1 allows us to disentangle the correlations documented in Tables 2 and 3, by showing children's cognitive test scores in subgroups defined by both parental ability and SEP. The gradients across SEP quintiles suggest that children whose parents are of low ability tend to do significantly better in cognitive tests if they are in a high SEP group (an average percentile rank of 51 in cognitive test scores for those in the top SEP quintile, compared to 37 in the bottom SEP quintile); however, there is no discernible SEP gradient for those whose parents are of middle and high ability. This could be because we only observe the cognitive ability of one parent. Low ability CMs who are in a high SEP group may be relatively likely to have high ability partners (which is unobservable in our data), and that may explain the better cognitive ability of their children.

Figure 1. Children's cognitive test scores by SEP and parental ability

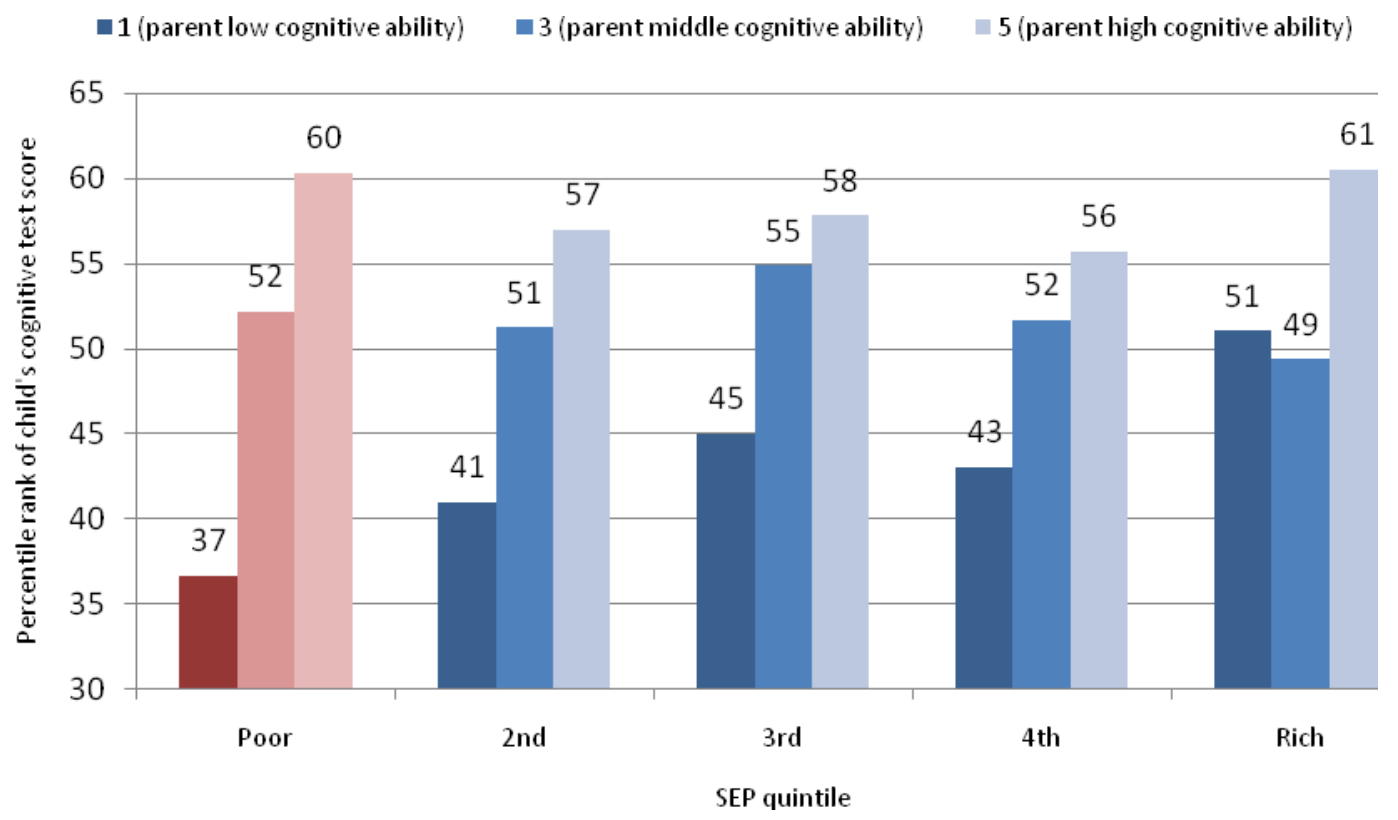

Notes: Authors' calculation using British Cohort Study. Our sample includes all children of the cohort members of the BCS aged between 3 and 16 in 2004 for whom we observe a BAS score. Parental ability refers to cognitive ability as measured when the parents were 10 years old, using BAS as well as additional tests of reading, vocabulary, writing, spelling, maths, copying, sentence formation and sequence recognition.

The clear upward gradient within SEP quintiles in Figure 1 shows that the correlation between parental abilities and children's abilities remains very strong after conditioning on SEP. Unless this conditional correlation is entirely explained by other observables (such as parental education), this implies that observing SEP but not parental ability would result in an over-estimate of the importance of SEP for cognitive skills (given the association between parental ability and SEP shown in Table 2). In the next section we investigate whether the association between SEP and/or parental ability and child cognitive skills holds once we control for a large set of additional covariates.

\section{Adding more parental characteristics} to the model: what difference does it make?

As explained, the fact that (unlike most studies of children's cognitive skills in the UK) we can account for detailed characteristics of one of the children's parents is of great significance. There is clear potential for these characteristics to be correlated both with our outcome of interest (the cognitive ability of children) and with typically observable explanatory variables (such as SEP and parental education). Therefore, when such characteristics are unobserved, they are likely to confound analyses that try to explain differences in 
cognitive skills amongst children. This has three particular consequences of interest here:

i. The role of parents' SEP in 'explaining' children's cognitive outcomes may be overstated: the correlation between the two may be due to the fact that high ability parents raise high ability children, and would do so irrespective of their socioeconomic status.

ii. When looking for possible transmission mechanisms, the extent to which the SEP gap in children's cognitive skills remains 'unexplained' by observable characteristics may also be overstated: controlling for parental ability and other information about the parent might allow us to 'explain away' more of the raw SEP gap. (This 'unexplained' component is sometimes interpreted as the 'direct effect' of SEP on cognitive ability.)

iii. The role of other factors in explaining the raw SEP gap may also be mis-stated: for example, the role of parental ability (or other parental characteristics), when it is not observed, may get attributed to observed factors with which it is correlated (e.g. parental education, attitudes, the home learning environment, and so on).

What difference does the additional information we have about the children's parents make to the kind of analysis of cognitive skills presented in the companion papers in this Special Issue? Here, we compare the results we get from the BCS data with and without using the information on parental cognitive ability, social skills and other characteristics that is not typically observed.

To do this we run two series of regressions, with children's cognitive test scores (age-normalised and in percentile ranks) as the dependent variable. In the first of these (shown in the upper panel of Table 4), we obtain the 'raw' SEP gap in children's test scores (shown in column 1 ) and sequentially add to the model parental education (column 4), demographics and other family background (column 5), and attitudes, educational aspirations, behaviours and the home-learning environment (column 6). These regressions are similar to those run in the other papers in this volume, which use information from a single generation only. The second series of regressions (shown in the lower panel of Table 4) differs only in that additional information from the parent's childhoods is also added to the model, before the other covariates (in columns 2 and 3). The difference between the two series of regressions is thus that the first one does not include columns 2 and 3: detailed information from the parent's childhood is never added to the model.

Column Regressors added to the model:

1) Current SEP quintile.

2) Parental cognitive ability.

3) Parental attitudes and social skills.

4) Parental education.

5) Demographics and family background.

6) Attitudes, educational aspirations, behaviours and the home-learning environment.

\section{Table 4. Children's cognitive test scores: regression results ${ }^{13}$}

\section{VARIABLES}

Using information from current generation only

Current SEP quintile $=2$

Current SEP quintile $=3$

Current SEP quintile $=4$

Current SEP quintile = 5 (richest)

Age mother left FT education: $17-18$ (relative to 16 or younger) Age mother left FT education: $19-22$ (relative to 16 or younger) Age mother left FT education: 23-25 (relative to 16 or younger) Age mother left FT education: 26 + (relative to 16 or younger) Father is self-employed (relative to full-time employee) Age of mother at birth of child: $20-24$ (relative to under 20) Age of mother at birth of child: 25-29 (relative to under 20) Age of mother at birth of child: $30-34$ (relative to under 20) 1 older siblings

2 older siblings

3 or more older siblings Twin

\section{(1)}

(2)

(3)

(4)

(5)

(6)

$3.4^{\star}$
$4.0^{\star \star}$
$6.4^{\star \star \star}$
$8.7^{\star \star \star}$
-
-
-
-
-
-
-
-
-
-
-

\begin{tabular}{|c|c|c|}
\hline $3.2^{*}$ & 2.4 & 0.8 \\
\hline $3.8^{\star \star}$ & $3.5^{\star}$ & 1.3 \\
\hline $6.1^{* \star \star}$ & $5.1^{\star \star}$ & 2.8 \\
\hline $7.3^{\star \star \star}$ & $6.6^{\star \star \star}$ & 3.4 \\
\hline $2.4^{*}$ & $3.8^{\star \star \star}$ & $2.2^{*}$ \\
\hline $4.2^{\star \star}$ & $6.4^{\star \star \star}$ & $4.3^{\star \star \star}$ \\
\hline 3.6 & $6.2^{\star \star}$ & 4.4 \\
\hline $9.0 * * *$ & $9.1^{\star \star \star}$ & 3.6 \\
\hline- & -2.4 & $-2.9^{\star \star}$ \\
\hline- & 1.4 & $5.4^{\star \star}$ \\
\hline- & $-14.2^{\star \star \star}$ & $5.5^{\star}$ \\
\hline- & $-18.3^{\star \star \star}$ & $6.6^{\star \star}$ \\
\hline- & $-6.3^{\star \star \star}$ & $-3.8^{\star * *}$ \\
\hline- & $-9.1^{\star \star \star}$ & $-6.2^{\star \star \star}$ \\
\hline- & $-13.2^{\star \star \star}$ & $-7.3^{\star \star}$ \\
\hline _ & $-13.9 * \star \star$ & $-14.1^{\star * *}$ \\
\hline
\end{tabular}


(Table 4 cont'd)

Breastfed for 6 months or more (relative to not breastfed)

Attends or attended playgroup

Parent thinks child likely to go to university or college

SDQ score (standardised)

Parental discipline score (standardised)

Parent-child conflict score (standardised, high score means more conflict)

Home-learning environment score for under-6s (standardised)

Harter perceived competence score (standardised, 10-16s only)

$\mathrm{CM}$ speaks to teacher about child at least once per term

Child goes to private school

Child can use a computer at home for homework (6-16-s only)

Child reads for enjoyment several times a week (6-16s only)

Child wants to continue studying post-16 (10-16s only)

Child has smoked (10-16s only)

Child (at least) sometimes drinks alcohol (10-16s only)

$4.4^{\star \star *}$

$2.4^{\star *}$

$13.2^{\star \star \star}$

$5.2^{\star \star \star}$

$2.8^{\star *}$

$1.9 * *$

$2.9^{* *}$

$-3.7^{* * *}$

$-3.4^{\star *}$

$7.3^{* *}$

$4.9 * *$

$9.6^{* \star *}$

$8.5^{\star * *}$

$-6.5^{\star *}$

$5.6^{* *}$

Including information about parent's childhood (including cognitive ability)

Current SEP quintile $=2$

Current SEP quintile $=3$

Current SEP quintile $=4$

Current SEP quintile = $\mathbf{5}$ (richest)

Age mother left FT education: $17-18$ (relative to 16 or younger) Age mother left FT education: $19-22$ (relative to 16 or younger) Age mother left FT education: 23-25 (relative to 16 or younger) Age mother left FT education: 26 + (relative to 16 or younger) Father is self-employed (relative to full-time employee) Age of mother at birth of child: $20-24$ (relative to under 20) Age of mother at birth of child: $25-29$ (relative to under 20) Age of mother at birth of child: 30-34 (relative to under 20) 1 older sibling

2 older siblings

3 or more older siblings

Twin

Breastfed for 6 months or more (relative to not breastfed)

Attends or attended playgroup

Parent thinks child likely to go to university or college

SDQ score (standardised)

Parental discipline score (standardised)

Parent-child conflict score (standardised, high score means more conflict)

Parent-child closeness score (standardised)

Home-learning environment score for under-6s (standardised) Harter perceived competence score (standardised, 10-16s only) CM speaks to teacher about child at least once per term

Child goes to private school

Child can use a computer at home for homework (6-16-s only)

Child reads for enjoyment several times a week (6-16s only)

Child wants to continue studying post-16 (10-16s only)

Child has smoked (10-16s only)

Child (at least) sometimes drinks alcohol (10-16s only)

$\mathrm{CM}$ thought they were good at maths, age 16

CM's quintile in cognitive test scores, age $5=2$

CM's quintile in cognitive test scores, age $5=3$

CM's quintile in cognitive test scores, age $5=4$

CM's quintile in cognitive test scores, age $\mathbf{5}=\mathbf{5}$ (brightest)

CM's quintile in cognitive test scores, age $10=2$

CM's quintile in cognitive test scores, age $10=3$

CM's quintile in cognitive test scores, age $10=4$

CM's quintile in cognitive test scores, age $10=5$ (brightest)

\begin{tabular}{|c|c|c|c|c|c|}
\hline $3.4^{*}$ & 1.6 & 1.5 & 1.6 & 1.3 & 0.2 \\
\hline 4.0 ** & 2.4 & 2.2 & 2.4 & 2.5 & 0.7 \\
\hline $6.4^{\star * *}$ & $4.3^{* *}$ & $3.9^{\star * \star}$ & $4.2^{\star \star}$ & $3.6^{*}$ & 2.1 \\
\hline $8.7^{\star \star \star}$ & $4.8^{\star \star}$ & $3.8^{\star *}$ & $3.7^{*}$ & 3.5 & 1.5 \\
\hline- & - & - & 1.4 & $2.9 * *$ & 1.8 \\
\hline- & - & - & 2.2 & $4.5^{\star \star}$ & $3.2^{\star}$ \\
\hline- & - & - & 0.9 & 3.7 & 3.1 \\
\hline- & - & - & $7.4^{\star \star *}$ & $7.2^{\star \star \star}$ & 2.8 \\
\hline- & - & - & - & $-2.5^{\star}$ & $-3.2^{\star \star}$ \\
\hline- & - & - & - & 0.3 & $4.6^{\star}$ \\
\hline- & - & - & - & $-15.7^{\star \star \star}$ & 5.0 \\
\hline- & - & - & - & $-20.1^{\text {*** }}$ & $5.8^{*}$ \\
\hline - & - & - & - & $-5.7^{\star \star \star}$ & $-3.2^{* * *}$ \\
\hline- & - & - & - & $-7.8^{\star \star \star}$ & -5.0 *** \\
\hline- & - & - & - & $-11.3^{\star \star *}$ & -5.7 \\
\hline - & - & - & - & $-13.5^{\star \star \star}$ & $-13.8^{\star \star \star}$ \\
\hline- & - & - & - & - & $3.1^{\star *}$ \\
\hline - & - & - & - & - & $2.4^{* *}$ \\
\hline- & - & - & - & - & $13.4^{\star \star \star *}$ \\
\hline- & - & - & - & - & $4.7^{\star \star \star}$ \\
\hline- & - & - & - & - & $2.6^{\star \star}$ \\
\hline - & - & - & - & - & $1.9 * \star$ \\
\hline- & - & - & - & - & -0.7 \\
\hline- & - & - & - & - & $2.7^{\star}$ \\
\hline- & - & - & - & - & $-3.4^{\star \star \star}$ \\
\hline - & - & - & - & - & $-3.6^{* * *}$ \\
\hline- & - & - & - & - & $8.3^{\star \star}$ \\
\hline- & - & - & - & - & $4.3^{\star *}$ \\
\hline- & - & - & - & - & $10.0^{* \star *}$ \\
\hline- & - & - & - & - & $8.3^{\star \star \star}$ \\
\hline- & - & - & - & - & $-6.9^{\star \star}$ \\
\hline- & - & - & - & - & $5.6^{\star \star}$ \\
\hline- & - & $4.0^{\star *}$ & $4.4^{\star \star}$ & $4.5^{\star \star}$ & $4.2^{\star \star}$ \\
\hline- & 3.2 & 3.1 & 2.8 & $3.7^{*}$ & $3.5^{\star \star}$ \\
\hline - & $7.3^{\star \star \star}$ & $6.8^{\star \star *}$ & $6.5^{\star \star \star}$ & $7.3^{\star \star \star}$ & $6.1^{\star \star *}$ \\
\hline - & 2.3 & 2.0 & 1.4 & 2.6 & 1.7 \\
\hline- & $7.2^{\star * *}$ & $6.6^{\star * *}$ & $6.2^{\star \star *}$ & $7.0^{* \star *}$ & $4.8^{* * *}$ \\
\hline- & $3.9^{*}$ & $3.5^{*}$ & 3.3 & 2.7 & $3.3^{*}$ \\
\hline- & $7.9 * \star \star$ & $7.3^{\star \star \star}$ & $7.1^{\star \star *}$ & $6.4^{\star \star \star}$ & $6.1^{\star \star \star}$ \\
\hline- & $10.3^{\star \star \star}$ & $9.2^{\star \star \star}$ & $9.1^{\star \star \star}$ & $9.0^{\star \star \star}$ & $9.2^{\star \star \star}$ \\
\hline- & $12.7^{\star \star \star}$ & $10.8^{* * *}$ & $10.3^{\star \star \star}$ & $10.8^{\star \star \star}$ & $10.6^{\star \star *}$ \\
\hline
\end{tabular}


Notes to Table 4: Authors' calculations using the British Cohort Study (BCS). Our sample includes all children of the cohort members of the BCS aged between 3 and 16 in 2004 for whom we observe a BAS score. Results reported are from a series of OLS regressions, robust to within-family clustering. One, two and three asterisks indicate statistical significance at the 10\%, 5\% and $1 \%$ level respectively. The covariates not listed here but that are included in the model (see Supplementary files for all coefficient estimates) are: father's education; the other behavioural and attitudinal information about the cohort member (i.e. one of the parents) as a child described in Section 2; social skills as measured by the Strengths and Difficulties Questionnaire; height and weight of the mother at birth; gender; ethnicity; whether a lone parent family; current health status of cohort member ('poor' to 'excellent'); whether cohort member currently has a long-term limiting illness; employment status of both the mother and father; age of mother at child's birth; number of older and younger siblings; whether a twin; whether attended nursery or playgroup; the home-learning environment (see Supplementary Files for details); whether child thinks good marks in education are important; whether they and their parent (the cohort member) want the child to continue in post-compulsory education; whether they and their parent think it is likely that the child will continue in post-compulsory education; whether they read for enjoyment; whether they have been suspended from school, smoked, broken the law, used cannabis, stolen from a store, bullied other children, been bullied, and been to youth clubs, scouts/guides or sports clubs/lessons at least once a week; whether school is private or single sex; whether parent (cohort member) thinks teaching at the school is 'very good'; whether child likes most of their teachers; levels of parental discipline, parent-child closeness and parent-child conflict (see Supplementary Files for details); whether the parent (cohort member) feels they spend enough time with their children; whether the family eats together every day; number of activities done as a family in last month; whether breastfed and how long for.

In Table 4 we present the coefficients for SEP and parental cognitive ability, as well as those coefficients that were statistically significant at the $5 \%$ level or above in the first series of regressions (without controlling for parental ability, social skills and attitudes) so that we can see whether they remain significant after controlling for the additional information that we observe about the parents. ${ }^{14}$ It is interesting to note that parental cognitive ability is the only factor observed during the parent's childhood which is statistically significant at the $1 \%$ level; conditional on other characteristics, social skills and attitudes and behaviours of the parents as children are not statistically significant (though they may have an effect that is mediated through the social skills, attitudes or other characteristics of their children).

Column 1 in Table 4 shows that children in the top SEP quintile are 9 percentiles higher up the cognitive test score distribution than those in the bottom SEP quintile, on average. This is the 'raw' SEP gap. This 'raw' gap is noticeably smaller than that present in the datasets analysed in the companion papers in this Special Issue. This may be because our sample is more homogeneous than those examined in the other papers, for at least two reasons: 1) all children in our estimation sample have at least one parent who was aged 31 or younger at the time of their birth; 2 ) the BCS is, by some distance, the oldest of the cohort studies considered, thus there has been considerably more time for attrition to occur since the first wave of analysis in 1970.

Columns 4-6 of the upper panel show how the residual SEP gap is reduced by controlling for the kinds of factors observed in the other papers in this Special Issue: parental education, demographics and other family background characteristics, and attitudes, educational aspirations, behaviours and the home-learning environment. Once we account for these, the residual SEP gap in cognitive skills is not statistically significant. This does not necessarily diminish the importance of SEP for cognitive development, as many of the factors we control for are plausible transmission mechanisms between SEP and cognitive skills.

If instead we control first for parental ability (column 2 of the lower panel), the residual SEP gap is reduced by 3.9 percentiles; controlling additionally for attitudes and social skills when the parents were children (column 3 ) reduces the residual gap by a further 1.0 percentile. ${ }^{15}$ This has the interesting implication that more than half of the raw SEP gap in children's cognitive skills, expressed in percentile ranks, can be predicted by one parent's cognitive ability and social skills, observed by the time that parent was 16 years old. Column 6 shows that, after adding all the controls, the residual gap between the top and bottom SEP quintiles is more than twice as large when we do not account for parental ability, attitudes and social skills (although this gap is not statistically significant in any case).

It is striking that, in contrast to SEP, the apparent importance of parental cognitive ability is relatively insensitive to the number of factors we control for (as shown by the coefficients on parental ability at the bottom of Table 4). We cannot rule out the possibility that there are unobserved covariates which would change this 
story if included in our model. However, given the very rich set of information we do observe, the results are at least suggestive of the fact that parental ability plays some kind of role in determining children's cognitive skills.

Reassuringly though, almost all of the statistically significant predictors of cognitive skills in the upper panel of Table 4 remain significant, and similar in magnitude, in the lower panel. This is (tentative) evidence that an inability to observe parental cognitive ability and other detailed information about the parents (as in the companion papers in this Special Issue, for example) may not grossly distort conclusions about which observable factors from the current generation are the most important predictors of cognitive skills. These factors include family structure (in particular, having older siblings and being a twin, strongly predict worse cognitive skills), the child's social skills and attitudes of both the parent and the child towards education.

To summarise, Table 4 suggests that, when unable to observe the detailed information about the children's parents that datasets like the BCS provide:

i) the (conditional) correlation between SEP and cognitive outcomes overstates the causal impact of SEP on cognitive outcomes;

ii) the 'unexplained' SEP gap is too large (although not statistically significant in our case), and an important part of the explanation for the raw SEP gap is therefore missed; but

iii) an assessment of which observed factors are the most important predictors of cognitive skills appears fairly robust to this omitted variable problem.

We investigate this further in the next section by quantifying the role of different factors in 'explaining' the SEP gap using decomposition analysis, both with and without accounting for the information we have from the BCS about the parents as children.

\section{Decomposing the SEP gap in children's cognitive test scores}

In this section, we compare in more detail the results from our final specification (that shown in column 6 in Table 4) obtained with and without controlling for parental ability, attitudes and social skills (as observed when the parents were children). To do this, we present results from two very simple decomposition analyses in Figure 2 . The decompositions show the fraction of the 'raw' cognitive gap between the top and bottom SEP quintiles that is attributable to other factors that we can observe. In the upper decomposition, these 'other factors' do not include parental ability, attitudes and social skills, while in the lower composition, these additional factors are included.

The contribution of each variable to the SEP gap is given by its coefficient estimate from the full regression specification multiplied by the extent to which it varies with SEP (the difference between the mean values of the variable in the top and bottom SEP quintiles). ${ }^{16}$ See Goodman, Gregg and Washbrook 2010 for more details. The variables have been grouped thematically for the purposes of the pie charts in Figure 2. The full list of variables in each group, along with coefficient estimates, conditional means and contributions to the raw SEP gap for the lower decomposition (including characteristics from the parents' own childhood) can be found in our Supplementary Files. 


\section{Figure 2. The SEP gap in cognitive test scores: decomposition analysis}

\section{Excluding parental cognitive ability, attitudes and social skills}

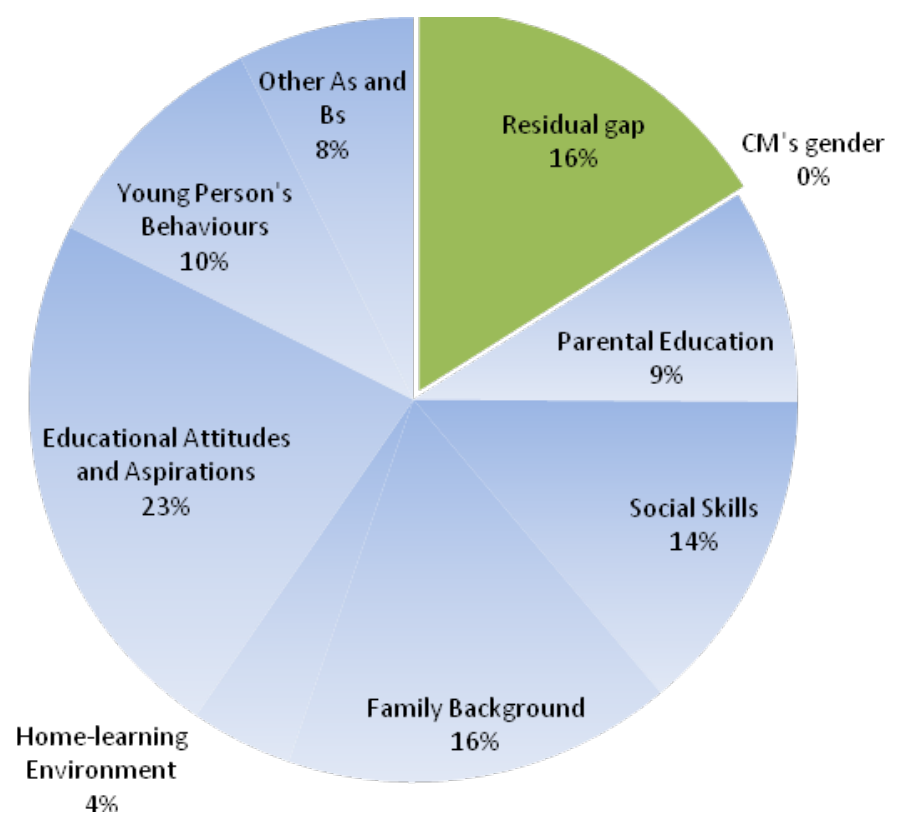

Including parental cognitive ability, attitudes and social skills

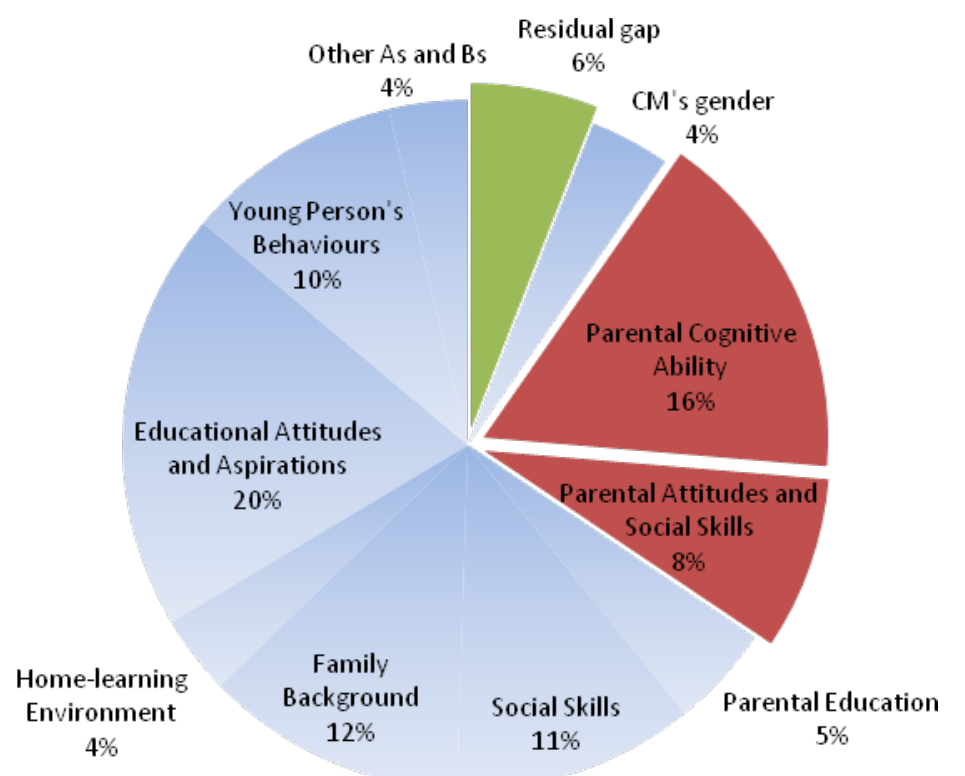

Notes: Authors' calculations using the British Cohort Study (BCS). Our sample includes all children of the cohort members of the BCS aged between 3 and 16 in 2004 for whom we observe a BAS score. The contribution of each variable to the SEP gap is given by the size of its conditional correlation with cognitive test scores (its coefficient estimate from the full regression specification) multiplied by the extent to which it varies with SEP (the difference between the mean values of the variable in the top and bottom SEP quintiles). Parental cognitive ability and parental attitudes and social skills were measured during the parent's childhood. Statistical significance of the coefficient estimates is not taken into account. 
Note that we exclude 'missing data' as a category in the decompositions, so the contribution of each group of variables in driving the SEP gap is presented as a percentage of the total contribution of all non-missing variables. This is because some information about the children of the BCS is observed only for children of certain ages (for example, self-reported attitudes and behaviours are observed only for those aged 10 or over), and is therefore subject to a lot of missing data when we group all children together in the analysis. This highlights that the decision to group children of all ages together is not trivial, in principle; however, the main conclusions we reach here are robust to disaggregating our sample of children by age.

The upper pie in Figure 2 shows that, of the variables from the current generation, the most important in accounting for the SEP gap in children's cognitive test scores are educational attitudes and aspirations (23\%), family background (16\%) and social skills (14\%). After accounting for everything that we observe in the current generation, $16 \%$ of the SEP gap remains unexplained.

The lower pie shows the importance of the additional information we have about the children's parents. These factors (shown in red) account for nearly one quarter of the raw SEP gap. Parental cognitive ability is by far the most important of these (16\%). Most noticeably, this reduces the unexplained component of the SEP gap to just 6\%. However, it does not change the relative importance of the factors observed during the current generation educational attitudes and aspirations, family background and social skills are still the most important of these - which, if our results have external validity, is reassuring for previous studies unable to account for parental ability.

Note that the indirect effects of parental cognitive ability may be very large. For example, more able parents may stay in education longer and have more positive attitudes towards the education of their children. The significant contribution of parental cognitive ability in Figure 2 is net of any indirect effects via the many covariates that we control for, such as the parents' educational outcomes and attitudes. In a similar decomposition based on a model including only SEP and parental cognitive ability, parental cognitive ability accounts for $50 \%$ of the gap in cognitive test scores between children from the richest and poorest SEP backgrounds. It thus seems likely that the true contribution of parental ability in explaining the SEP gap in cognitive skills, accounting for both direct and indirect effects, lies somewhere in the range $16-50 \%$.

In addition, we could conjecture that higher parental ability increases children's cognitive skills by making the time spent on children's cognitive development more productive (e.g. reading with one's children may be more productive if the parent is a good reader). However, the importance of parental cognitive ability does not seem to be largely driven by a complementarity with observed aspects of parenting, in as much as most interaction terms between parental cognitive ability and environmental factors are not statistically significant when added to the model (not shown). ${ }^{17}$ Thus, although our findings are based on a simple linear regression model in which endogeneity cannot be ruled out, we interpret the large contribution of parental cognitive ability in Figure 2 - which remains even after controlling for a very wide range of plausible transmission mechanisms between parental and child cognitive ability - as being suggestive of the possibility of some kind of genetic link between the cognitive skills of parents and their children, although we acknowledge that the interactions between genetics and the environment are necessarily complex.

Finally, since we only have detailed information about one of the parents (the cohort member) of the children in our sample, assortative mating (the tendency for people to have partners with characteristics similar to themselves) may obscure one reason why cleverer parents raise cleverer children - namely, that they have cleverer partners. Since we observe many family-level environmental factors (such as the home-learning environment), we reduce the scope for us to confound the importance of parental ability with the importance of the role played by the CM's partner in providing a home environment conducive to cognitive development. However, we are not able to fully account for the likelihood that high cognitive ability of the $\mathrm{CM}$ is associated with high cognitive ability of the CM's partner (we may partially account for it because we observe the education levels of both parents, which should proxy cognitive ability). We are therefore likely to be picking up the combined effect of some kind of genetic transmission from both parents. 


\section{Conclusion}

Studies of children's cognitive abilities typically relate them to the cognitive abilities of their parents or to socio-economic and attitudinal factors (all of which tend to correlate). In this paper, we have made use of a dataset which unusually allows us to do both simultaneously. This has enabled us to consider the intergenerational transmission of cognitive abilities and the rich-poor gap in cognitive abilities as a coherent whole. In particular, it has allowed us to deal directly with a potentially serious omitted variables problem that could confound many studies of the relationship between childhood poverty and children's outcomes (as well as studies that seek to identify potential transmission mechanisms between the two).

In line with other studies, we have found that parental cognitive ability is a very important predictor of children's cognitive skills (the most important, along with educational attitudes and aspirations). Because parents' cognitive ability and socio-economic position are in turn also very strongly related, studies which examine the relationship between parental SEP and children's ability, but do not control for parental ability - or in some other way attempt to account for these factors - will suffer from a serious omitted variables problem. Indeed, our estimates suggest that parental cognitive ability accounts for 16 percent of the gap in cognitive test scores between children from rich and poor families after controlling for a wide range of mechanisms through which ability may be transmitted across generations (for example, differences in the home learning environment that parents provide for their children), and 50 percent of the gap if we do not include such factors.

This finding raises important questions about the relevance of genetic inheritance in accounting for the gap in cognitive test scores between children from rich and poor families. This is clearly a controversial and complex topic. Our results do not suggest the strong complementarities between parental ability and other observed aspects of parenting that we had expected to find, and are tentatively suggestive of an important - albeit likely complex - role for genetic inheritance.

Interestingly, however, the inclusion of parental ability within our model does not substantively alter our impression of which of the other determinants of cognitive skills are important: for example, family structure, the child's social skills, and attitudes of both the child and the parent towards education are all important predictors of children's ability, even after taking parental ability into account. This is reassuring for studies such as the others in this volume, which are not able to control for parental ability, but find an important role for characteristics such as parental attitudes to education, the home learning environment, and children's behaviours and social skills as potential transmission mechanisms between parental SEP and children's cognitive outcomes.

\section{Acknowledgements}

This work was carried out as part of a larger body of work funded by the Joseph Rowntree Foundation (JRF) through its programme of research on education and poverty. We gratefully acknowledge the support of JRF, and the generous and productive guidance of Helen Barnard and Chris Goulden. We are also thankful to members of the advisory group: Tim Crosier, Naomi Eisenstadt, Leon Feinstein, Zoe Ferguson, Tony Foot, Leslie Gutman, Lisa Harker, Andrew Ledger, Mark Newman, Ivan Turok, Anna Vignoles, Jane Waldfogel, and Stephen Witt. All errors remain the responsibility of the authors.

\section{References}

Anger S and Heineck G. (2010) Do Smart Parents Raise Smart Children? The Intergenerational Transmission of Cognitive Abilities. Journal of Population Economics , 23, 1255-1282.

Björklund A, Hederos Eriksson K and Jäntti M. (2010) IQ and family background: are associations strong or weak? Berkeley Electronic Journal of Economic Analysis and Policy 10.

Black S, Devereux P and Salvanes K. (2009) Like father, like son? A note on the intergenerational transmission of IQ scores. Economic Letters, 105, 138-140.

Blanden J, Gregg P and Macmillan L. (2007) Accounting for Intergenerational Income Persistence: Noncognitive Skills, Ability and Education, Economic Journal, 117, C43-C60.

Blau D. (1999) The Effect of Income on Child Development, Review of Economics and Statistics, 81, 261-276. 
Carneiro P, Crawford C and Goodman A. (2007) The impact of early cognitive and non-cognitive skills on later outcomes. CEE Discussion Paper No. 92.

Conners CK. (1969) A teacher rating scale for use in drug studies. American Journal of Psychiatry, 126, 884888.

Cunha F and Heckman J. (2007) The technology of skill formation. American Economic Review, 97, 31-47.

Dahl G and Lochner L. (2008) The Impact of Family Income on Child Achievement: Evidence from the Earned Income Tax Credit. NBER Working Paper No. 14599.

Davis-Kean P. (2005) The influence of parent education and family income on child achievement: the indirect role of parental expectations and the home environment. Journal of Family Psychology, 19, $294-304$.

Dickens W and Flynn J. (2001) Heritability estimates versus large environmental effects: The IQ paradox resolved. Psychological Review, 108, 346-369.

Dickens W and Flynn J. (2002) The IQ paradox: Still resolved. Psychological Review, 109, 1-25.

Duflo E. (2000) Child Health and Household Resources in South Africa: Evidence from the Old Age Pension Program. American Economic Review, 90, 393-398.

Duncan G, Morris P and Rodrigues C. (2006) Does Money Really Matter? Estimating Impacts of Family Income on Young Children's Achievement with Data from Random-Assignment Experiments. https://www.earlyadolescence.org/files/IOM/Does\%20Money\%20Really\%20Matter Restatrev FINA L 123 2006.pdf

Duncan G, Young W, Brooks-Gunn J and Smith J. (1998) How much does childhood poverty affect the life chances of children? American Sociological Review, 63, 406-423.

Duncan G and Brooks-Gunn J. (1997) Consequences of Growing Up Poor. Russell Sage Foundation, New York.

Ermisch J, Francesconi M and Pevalin D. (2002) Childhood parental behaviour and young people's outcomes. ISER Working Paper 2002-12, University of Essex.

Goodman A, Gregg P and Washbrook L. (2010) Children's educational attainment and the aspirations, attitudes and behaviours of parents and children through childhood. Longitudinal and Life Course Studies, 2, 1-18.

Guo $G$ and Harris K. (2000) The mechanisms mediating the effects of poverty on children's intellectual development. Demography, 37, 431-447.

Heckman J, Stixrud J and Ursua S. (2006) The Effects of Cognitive and Noncognitive Abilities on Labor Market Outcomes and Social Behavior. Journal of Labor Economics, 24, 411-482.

Jean Yeung W, Linver M and Brooks-Gunn J. (2008) How money matters for young children's development: parental investment and family processes. Child Development, 73, 1861-1879.

Levy D and Duncan G. (2000) Using Sibling Samples to Assess the Effect of Childhood Family Income on Completed Schooling. JCPR Working Papers, Northwestern University/University of Chicago Joint Center for Poverty Research.

Loehlin J. (2002). The IQ paradox: Resolved? Still an open question. Psychological Review, 109, $754-758$.

Mayer S. (1997) What Money Can't Buy: Family Income and Children's Life Chances. Harvard University Press, Cambridge, MA.

Rowe D and Rodgers J. (2002) Expanding variance and the case of historical changes in IQ means: A critique of Dickens and Flynn (2001). Psychological Review, 109, 759-763.

Sacerdote B. (2002) The Nature and Nurture of Economic Outcomes. American Economic Review, 92, 344348.

Sacerdote B. (2007) How Large Are the Effects from Changes in Family Environment? A Study of Korean American Adoptees. Quarterly Journal of Economics, 122, 119-157.

Shea J. (2000) Does parents' money matter? Journal of Public Economics, 77, 155-184.

\section{Endnotes}

\footnotetext{
${ }^{1}$ Originally called the British Births Survey (BBS), the birth survey covered the whole of the United Kingdom, but those from Northern Ireland were dropped for subsequent sweeps.

${ }^{2}$ Children aged 10 or over were given an additional questionnaire of their own to complete.

${ }^{3}$ The British Ability Scales comprise a mixture of measures of educational attainment, including number skills, spelling and reading, and measures of cognitive abilities that are the outcome of interactions between a child's innate capabilities and his or her experiences, both at home and at school. For more details, see: http://www.glassessment.co.uk/health_and_psychology/resources/british ability scales/british ability scales.asp?css=1.

${ }^{4}$ See http://www.ifs.org.uk/publications/5269 for a full list of variables included in our analysis.

${ }^{5}$ See www.cls.ioe.ac.uk/core/documents/download.asp?id=932\&log_stat=1 for more details.
} 
${ }^{6}$ There is also a myriad of other information about the parents' childhood that we could have included in our analysis (including SEP). However, the inclusion of these other factors did not materially affect our results, so for the sake of brevity, we focus our attention on cognitive ability, social skills and some selected attitudes to education and future careers.

${ }^{7}$ This means we take account of income in the age 29 wave as well as the age 34 wave.

${ }^{8}$ Precisely, all children have at least one parent who is 34.

${ }^{9}$ Indeed, the only directions of causality we could rule out a priori would be parental ability being affected by either of the other two (since we observe parental ability during the parent's childhood).

${ }^{10}$ Of course, parental ability may not be the only latent factor that can lead to omitted variable bias. The same could be true of other characteristics of the parents which we also (unusually) observe in the BCS and are able to account for in this paper, such as their attitudes or social skills. For example, parents that are more motivated may end up in higher paying jobs and may also provide greater stimulation for their children (leading to higher cognitive test scores).

${ }^{11}$ Note that SEP and parental cognitive ability are measured continuously but we have categorised them into quintiles here. This means, for example, that children in the same parental ability quintile who are in very different SEP quintiles are relatively likely to be far apart within the parental ability quintile (or vice versa). An association between parental ability and cognitive skills would then be obscured by our discrete categorisation, and would be attributed to SEP instead (or vice versa). Therefore, the precise gradients in Figure 1 depend upon how crudely we disaggregate the $\mathrm{SEP} /$ parental ability distributions, which is arbitrary. Our qualitative conclusions are robust to this choice, however: continuous measures of SEP and parental ability are both statistically significant predictors of cognitive ability when controlled for simultaneously in an OLS regression, but the $p$-value for parental ability is much lower than that for SEP; and the tendency for SEP to be more strongly associated with cognitive skills where parents are low ability is also statistically significant (confirmed by adding an interaction between SEP and an indicator variable equal to one if the parent is below the median of the ability distribution).

${ }^{12}$ The association between children's and parents' ability varies by gender, as found by Anger and Heineck (2010) using German data. It is strongest where the parent and child are the same sex (a correlation of 0.23 for fathers and sons and 0.22 for mothers and daughters), and lowest of all for fathers and daughters, with a correlation of 0.13 . These correlations are quite low compared to others in the literature. For fathers and sons, Bjorklund et al 2010, obtain a correlation of 0.35 for Sweden, which closely matches estimates from Black et al 2009, for Norway. The Anger and Heineck study, looking at parents and children of all genders, found a correlation of 0.5 . Possible explanations for these different correlation coefficients include: different measures of cognitive ability; data from different countries and different cohorts (for example, the parents in our sample were born nearly 40 years later than those used by Black et al); and non-random sample selection in our study and (to varying extents) the other studies.

${ }^{13}$ We have replicated this regression with SEP quintiles replaced by income quintiles, to test the sensitivity of our results to our measure of material wellbeing. The coefficients on income are notably smaller than those on SEP (the raw gap between the top and bottom quintiles is 6.2 percentile ranks, rather than 8.7 ) - this could reflect measurement error in income - but the raw gaps are still statistically significant at the $1 \%$ level. Coefficient estimates on other variables are virtually unaffected.

${ }^{14}$ All coefficient estimates from column 6 of the lower panel of Table 4 can be found in our Supplementary Files: a full set of results is available from the authors on request.

${ }^{15}$ As explained, the variables that we loosely refer to as parental characteristics are more precisely the characteristics of one of the children's parents (the cohort member). Exceptions are education and employment status, which we observe for both parents.

${ }^{16}$ Note that the 'contribution' of a variable to the SEP gap says nothing about statistical significance: it depends on the magnitude of estimated coefficients but not the precision with which they are estimated.

${ }^{17}$ Specifically, we created an indicator variable that equals one if the parent was in the top half of the cognitive ability distribution at age 10, and interacted this with variables capturing: parental discipline, parent-child closeness, parentchild conflict, a composite home learning environment score, the number of activities done together as a family per week, whether the parent reads to the child daily, whether the parent regularly attends parents' evenings, whether the parent feels they spend enough time with their children, whether the parent helps with homework and whether the parent makes sure homework is completed. These interactions are jointly insignificant at the $10 \%$ level. 